\title{
PEG10 promotes human breast cancer cell proliferation, migration and invasion
}

\author{
XINRAN LI $^{1 *}$, RUIJING XIAO ${ }^{1 *}$, KINGSLEY TEMBO $^{1}$, LING HAO $^{1}$, MENG XIONG $^{1}$, \\ SHAN PAN $^{1}$, XIANGYONG YANG ${ }^{2}$, WEN YUAN ${ }^{1}$, JIE XIONG $^{1}$ and QIUPING ZHANG ${ }^{1}$ \\ ${ }^{1}$ Department of Immunology, School of Basic Medical Sciences, Wuhan University, Wuhan, Hubei 430071; \\ ${ }^{2}$ Hubei University of Technology Engineering and Technology College, Wuhan, Hubei 430068, P.R. China
}

Received December 14, 2015; Accepted January 8, 2016

DOI: $10.3892 /$ ijo.2016.3406

\begin{abstract}
Paternally expressed imprinted gene 10 (PEG10), derived from the Ty3/Gypsy family of retrotransposons, has been implicated as a genetic imprinted gene. Accumulating evidence suggests that PEG10 plays an important role in tumor growth in various cancers, including hepatocellular carcinoma, lung cancer and prostate cancer. However, the correlation between PEG10 and breast cancer remains unclear. In the present study, we evaluated and characterized the role of PEG10 in human breast cancer proliferation, cell cycle, clone formation, migration and invasion. The expression level of PEG10 was significantly elevated in breast cancer tissues and associated with distant metastasis and poor clinical outcome. Gene set enrichment analysis indicated that high expression of PEG10 could enrich cell cycle-related processes in breast cancer tissues. Ectopic overexpression of PEG10 in breast cancer cells enhanced cell proliferation, cell cycle, clone formation along with migration and invasion. Cell-to-cell junction molecule E-cadherin was downregulated and matrix degradation proteases MMP-1, MMP-2, MMP-9 were upregulated after PEG10 overexpression. Our results demonstrated that PEG10 is a crucial oncogene and has prognostic value for breast cancer, which could be applied in breast cancer diagnosis and targeting therapy in future.
\end{abstract}

Correspondence to: Professor Qiuping Zhang or Dr Jie Xiong, Department of Immunology, School of Basic Medical Sciences, Wuhan University, 185 Donghu Road, Wuchang, Wuhan, Hubei 430071, P.R. China

E-mail: qpzhang@whu.edu.cn

E-mail: jiexiong@whu.edu.cn

*Contributed equally

Abbreviations: PEG10, paternally expressed imprinted gene 10; GEO, Gene Expression Omnibus; GSEA, gene set enrichment analysis; MMPs, matrix metalloproteinases; TIMPs, tissue inhibitor of metalloproteinases

Key words: PEG10, breast cancer, proliferation, migration, invasion

\section{Introduction}

Breast cancer ranks as the second cause of cancer death among women (1-4). Clinical cases have indicated that most of the breast cancer deaths are caused by metastasis $(5,6)$, and as a distinct metastatic pattern, breast cancer cells are more inclined to migrate into lymph nodes, bone marrow, lung, liver and brain (7). Extensive efforts have been made towards a better understanding of human breast cancer oncogenic events, and many biomarkers have been reported to be an indicator for initiation and invasion of breast cancer. These may include among others; proline rich inositol polyphosphate 5-phosphatase (PIPP), which when depleted inhibits PI3K/AKT signaling, enhanced the transformation ability of breast cancer cells, but reduced cell migration and invasion thus indicating that PIPP is a potential suppressor of oncogenic PI3K/AKT signaling in breast cancer (8); bone marrow stromal antigen 2 which is expressed in cancer cells and facilitates the emergence of neoplasia and malignant progression of breast cancer (9) and others such as oncoprotein hepatitis B X-interacting protein which when mediated by general control non-derepressible 5 promotes the migration of breast cancer cells and therapeutically could act as a novel target in breast cancer (10).

PEG10 (paternally expressed imprinted gene 10) located at human chromosome $7 \mathrm{q} 21$ was first reported to a gene from a newly defined imprinted region in 2001 (11). Akamatsu et al have reported that PEG10 was highly expressed in neuroendocrine prostate cancer (NEPC), and distinct isoforms of PEG10 promoted proliferation and invasion of NEPC cells, which could be recognized as a specific therapeutic target for NEPC (12). Abnormal overexpression of PEG10 was found in most hepatocellular carcinoma cells, and regenerating mouse liver cells (13). PEG10 expression was reported to be associated with worse survival and recurrence in hepatocellular carcinoma, which could indicate that PEG10 as a potential target predicting early recurrence and recurrence-free survival after curative resection (14). Long-term inhibition of PEG10 was found to lead to induction of apoptosis in B-cell chronic lymphocytic leukemia (B-CLL) cells (15). The PEG10 knockout mice showed early embryonic lethality owing to defects in the placenta, which indicated that PEG10 played an important role in placental 
development (16). PEG10 expression was closely associated with the clinical, pathological and biological behaviors, and a poor prognosis in gallbladder cancer (17). In breast cancer transgenic mouse models with c-MYC overexpression, most of the mammary carcinomas developed by overexpressing c-MYC had increased PEG10 mRNA expression compared with normal mammary gland (18). Though PEG10 has been implicated in many types of cancers, the function of PEG10 in human breast cancer still remains unclear.

In recent years, we have focused on PEG10 in several cancers, including hematological malignancies and lung cancer. We have observed that in $\mathrm{CD} 19^{+} \mathrm{CD} 34^{+} \mathrm{B}$ cells from patients with B-cell lineage acute (B-ALL) and B-CLL, PEG10 was activated by CXCR 5 and CCR7 co-simulation thus contributing to apoptosis resistance (19). Similarly in B-ALL CD23 ${ }^{+} \mathrm{CD}^{+} \mathrm{B}$ cells, PEG10 expression level was upregulated by CCL19 and CXCR13, which also enhanced the anti-apoptosis ability (20). PEG10 promoted Raji cell migration capacity by up-regulating matrix metalloproteinase- 2 (MMP-2) and MMP-9 expression (21). Recently, we observed that the expression of PEG10 was closely related to clinical TNM grade and patient prognosis in lung cancer through publicly available datasets. Amplifying this finding, in vitro experiments in A549 cells, indicated that PEG10 facilitated cell proliferation and promoted tumor cell migration and invasion by upregulating the expression of $\beta$-catenin, MMP-2 and MMP-9 (22). Therefore using Gene Expression Omnibus (GEO) datasets, we analyzed the relationship between PEG10 and breast cancer, and found that the high expression of PEG10 was positively correlated with the poor grade of breast cancer and closely related with overall survival of the patients. Thus, we hypothesize that the elevation of PEG10 also enhances breast cancer cells proliferation and invasion.

\section{Materials and methods}

Cell culture. Human breast cancer cell line MDA-MB-231 and human hepatoma cell line HepG2 were obtained from American type culture collection (ATCC) and maintained in Dulbecco's modified Eagle's medium (DMEM) with high glucose (Gibco, Carlsbad, CA, USA) supplemented with 10\% fetal bovine serum (FBS; Hyclone Corp., Logan, UT, USA), containing $100 \mathrm{U} / \mathrm{ml}$ penicillin and $100 \mathrm{mg} / \mathrm{ml}$ streptomycin. Cells were cultured in a $5 \% \mathrm{CO}_{2}$ air incubator at $37^{\circ} \mathrm{C}$ and passaged using $0.25 \%$ trypsin-EDTA (Gibco) when they reached confluence.

Plasmid and transfection. Full-length PEG10 was isolated from HepG2 cells, and then subcloned into pEGFP-c1 plasmid between HindIII and SalI restriction sites. The orientation and correct frame of the recombinant vector pEGFP-c1/ PEG10 (PEG10-EGFP) and the empty vector pEGFP-c1 plasmid used as control (Control-EGFP) were confirmed by sequencing.

For transient transfection, cells were first seeded in plate at $60 \%$ confluency $\left(5 \times 10^{5}\right.$ cells per well) overnight. The next day plasmids were diluted with an appropriate concentration of Opti-MEM according to manufacturer's instruction of Lipofectamin ${ }^{\mathrm{TM}} 3000$ to form complexes. The cells were transiently transfected with PEG10-EGFP plasmid or Control-EGFP plasmid complexes for $48 \mathrm{~h}$. The viability of the cells was tested by trypan blue staining.

Cell proliferation assay. MDA-MB-231 cells were seeded in a 96-well plate at the density of $1 \times 10^{4}$ cells per well overnight for adherence. The next day cells were transfected with the above mentioned plasmids. After $48 \mathrm{~h}$ of transfection, $10 \mu \mathrm{l}$ CCK-8 solutions was added to each well at indicated times and incubated for another $3 \mathrm{~h}$. The absorbance of each well was obtained by PerkinElmer 2030 VICTOR X Multilabel Plate Reader (Perkin-Elmer, Waltham, MA, USA) at $450 \mathrm{~nm}$.

Cell cycle. Cells were transfected for $48 \mathrm{~h}$ and digested by trypsin. After incubation with $70 \%$ ethanol overnight, cells were stained with propidium iodide (PI) for $30 \mathrm{~min}$. The cells were then washed with ice cold PBS twice and PI intensity was detected by flow cytometry. The results were analyzed using ModFit software as directed by manufacturer's specifications.

Wound-healing assay. Confluent monolayers of MDA-MB231 cells $(60 \%)$ were triple plated in a 24 -well plate, and transfection was done as described above. After transfection for $48 \mathrm{~h}$, cells were starved with DMEM solution containing $1 \%$ FBS for $24 \mathrm{~h}$. A wound was then scratched in each well using a sterile P10 micropipette tip. Cells were allowed to migrate for $24 \mathrm{~h}$ and images were obtained by microscopy using a charge coupled device (CCD) camera. All analyses were repeated in triplicate.

Transwell migration and Matrigel invasion assay. Cells were transfected with plasmids for $48 \mathrm{~h}$ as described above. The cells were then digested, centrifuged and suspended into DMEM medium with $1 \%$ FBS. A total of $5 \times 10^{4}$ PEG10-EGFP and Control-EGFP cells were plated in upper chamber, which was a $6.5-\mathrm{mm}$-diameter, polycarbonate membrane with an $8-\mu \mathrm{m}$ pore size filter, coated (invasion) or not coated (migration) Matrigel (Corning, NY, USA). The lower chamber was filled with $500 \mu$ l DMEM solution containing $20 \%$ FBS, which acted as a chemoattractant. After $24 \mathrm{~h}$ of incubation at $37^{\circ} \mathrm{C}$, non-migrated cells were removed with cotton swabs and migrated cells were fixed in methanol and stained with $0.1 \%$ crystal violet. Images were obtained by light microscope with charge coupled device camera. Cells from four different fields were counted.

Clone formation assay. Transfected cells (500) were seeded to each well of 6-well plate and allowed to grow for 10 days in $37^{\circ} \mathrm{C}$ incubator. The cells were then washed twice with ice cold PBS, fixed by methanol for $10 \mathrm{~min}$ and stained with crystal violate for $10 \mathrm{~min}$. The images of stained and clone spheres of cells were obtained by a charge coupled device camera.

$R N A$ extraction and real-time quantitative RT-PCR. Total RNA was extracted from MDA-MB-231 cells using TRIzol (Invitrogen, USA) according to the manufacturer's specifications and quantified by NanoDrop 2000 (Thermo Scientific, Waltham, MA, USA). RNA ( $2 \mu \mathrm{g})$ was reverse-transcribed to cDNA with random primers using the reverse transcriptase kit 



Figure 1. PEG10 was highly expressed in breast cancer and closely correlated with clinicopathological features. Analysis of PEG10 expression in normal breast and breast carcinoma in GSE42568 (A) $(* \mathrm{*} p<0.005)$ and GSE10780 (B) $(* * * * 00.001)$, respectively. PEG10 expression in different grade of breast cancer progression in GSE42568 (C) ( $(* \mathrm{p}<0.005)$. Tumor sizes were analyzed in low expression and high expression of PEG10 groups in GSE4922 (D) ( $\mathrm{p}<0.05)$. PEG10 expression in heathy, DCIS and IDC groups in GSE21422 (E) ( $\left.{ }^{*} \mathrm{p}<0.05\right)$. PEG10 expression in non-metastasis and metastasis groups in GSE42568 (F) $(\mathrm{p}<0.05)$. DICS, ductal cancer in situ; IDC, invasive ductal carcinomas; metastasis here defined as lymph node metastasis.

(Promega, Madison, WI, USA) according to manufacturer's protocol. The sequences of specific primers were as follows: PEG10: Forward: 5'-GCTAAGCTTCGATGACCGAACG AAGAAGGGACGAG-3', Reverse: 5'-ATATAGTCGACC TACAGCGGGGCCGGGGAGTTT-3'; MMP-1: Forward: 5'-GGGGCTTTGATGTACCCTAGC-3', Reverse: 5'-TGTCA CACGCTTTTGGGGTTT-3'; MMP-2: Forward: 5'-TGACA TCAAGGGCATTTCAGGAGC-3', Reverse: 5'-GTCCGCC AAATGAACCGGTCCTTG-3'; MMP-9: Forward: 5'-GAGG TTCGACGTGAAGGCGCAGATG-3', Reverse: 5'-CATA GGTCACGTAGCCCACTTGGTC-3'; TIMP-1: Forward: 5'-CTTCTGCAATTCCGA CCTCGT-3', Reverse: 5'-ACGCT GGTATAAGGTGGTCTG-3'; TIMP-2: Forward: 5'-GCTGC GAGTGCAAGATCAC-3', Reverse: 5'-TGGTGCCCGTT GATGTTCTTC-3'; E-cadherin: Forward: 5'-ATTTTTCCCT CGACACCCGAT-3', Reverse: 5'-TCCCAGGCGTAGACC AAGA-3'; GAPDH: Forward: 5'-CTGGGCTACACTGAGC ACC-3', Reverse: 5'-AAGTGGTCGTTGAGGGCAATG-3';

Protein extraction and western blotting. Transfected cells were washed with ice cold PBS and lysed by RIPA supplemented with protease inhibitor PMSF on ice. Concentrations of total protein were measured by BCA kit (Thermo Scientific) and absorbance was obtained by the PerkinElmer 2030 VICTOR X Multilabel Plate Reader. The extracted proteins were separated by $10 \%$ SDS-polyacrylamide gel electrophoresis and transferred to PVDF membranes (Millipore, Billerica, MA, USA). The membrane were blocked with $5 \%$ non-fat milk TBS-T $(0.1 \%$ Tween-20, $100 \mathrm{nM}$ Tris- $\mathrm{HCl}, 0.9 \% \mathrm{NaCl})$ and incubated with primary antibodies with gentle shaking at $4^{\circ} \mathrm{C}$ overnight. After washing four times, the membranes were incubated with HRP-conjugated secondary antibodies for $2 \mathrm{~h}$. The signals were detected using an enhanced chemiluminescence detection kit (Thermo Scientific).

Bioinformatics analysis. Several GEO datasets were used to analyze the correlations of PEG10 and clinical pathological features. PEG10 expression was traced by probe 212092 and 212094 in the platform. The PEG10 expression of different groups were obtained and compared by two-tail t-test. In order to observe the overall survival, patients were divided into PEG10-high-expression (top 25\%) and PEG10-lowexpression group (remaining 75\%) and survival curves were obtained by Kaplan-Meier and Log-rank tests which could 
A

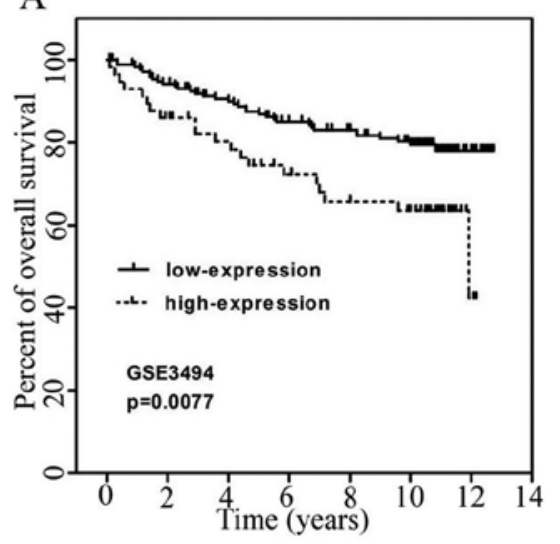

$\mathrm{C}$



$\mathrm{D}$



B



$\mathrm{E}$

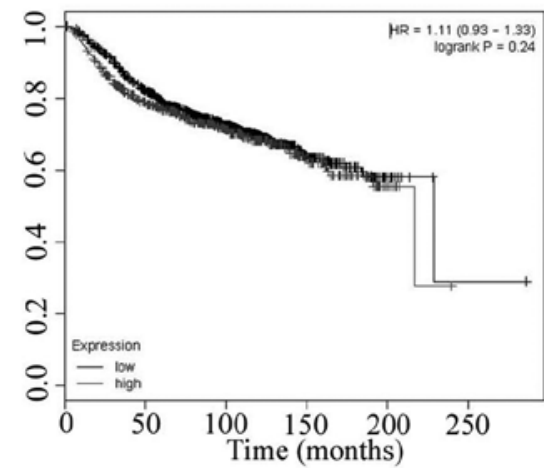

Figure 2. Upregulation of PEG10 led to a poor outcome of breast cancer patients. Kaplan-Meier analyses were performed in PEG10-high group and PEG10-low group with GSE3494 (A) and GSE42568 (B) to investigate the relationship between PEG10 expression and overall survival rate. The correlation of PEG10 and survival rate in total samples (C), in lymph node metastasis patients (D) and in non-metastasis patients (E) were determined using www.kmplot.com with auto selected best cut-off.

also be obtained using www.kmplot.com website with auto select best cutoff for dividing group. In addition, for Gene Set Enrichment Analysis (GSEA), GSE3494 dataset was analyzed with GMT file C5 (GO gene set) as directed by the manufacturer's specifications.

Statistics. Statistical analysis of significant differences were acquired using the GraphPad Prism 5 software. Significance was assessed by t-test followed by unpaired comparisons. ANOVA test was used to evaluate PEG10 expression variation in different tumor grades. Kaplan-Meier plots were constructed and log-rank tests were used to observe overall survival rates related to PEG10 expression. A probability (P) value of $\leq 0.05$ was considered to indicate a statistically significant difference.

\section{Results}

PEG10 was highly expressed in breast cancer and closely correlated with clinicopathological features. From GEO database, we applied the t-test to analyze several datasets containing breast cancer samples with or without normal breast tissues and corresponding clinical information. PEG10 expression was measured as $\log _{2}$ (probe intensities) using Affymetrix microarrays. As shown in Fig. 1A, PEG10 expression was notably elevated in breast carcinoma compared to normal breast samples (GSE42568, $\mathrm{p}=0.0049$ ). Similar results were obtained in GSE10780 dataset indicating that PEG10 was expressed highly in breast cancer compared to normal tissues (Fig. 1B, p=0.0002). High PEG10 expression was related to high grade of breast cancer (Fig. 1C, GSE42568, p=0.006). From GSE4922 dataset, PEG10 high expression was correlated with tumor size and presented more heterogeneity than low-level group (Fig. 1D, p=0.0333). In addition, patients with invasive ductal carcinomas (IDC) (GSE21422, Fig. 1E, $\mathrm{p}=0.039$ ) and lymph node metastasis (GSE42568, Fig. 1F, $\mathrm{p}=0.023)$ were likely to show PEG10 high expression.

Upregulation of PEG10 led to a poor outcome of breast cancer patients. Consistent with above pathological observations, we analyzed the GEO datasets GSE3494 and GSE42568 to investigate the relationship between PEG10 expression and overall survival rate. According to PEG10 expression, top 25\% of samples were considered as the PEG10 high-expression group, and $75 \%$ of the samples were included in the PEG10 lowexpression group. The Kaplan-Meier method and log-rank test were used to compare the survival rate of patients with breast cancers in the two groups described above. The data indicated that patients in high-expression group present poorer survival rates than the low-expression group in GSE3494 (Fig. 2A, $\mathrm{p}=0.0077$ ) and GSE42568 (Fig. 2B, p=0.0496). Similar results were observed using www.kmplot.com with auto selected best cutoff in which PEG10 high group have lower survival rates with $p$ value of 0.016 (Fig. 2C). In addition, survival rate of patients with high PEG10 expression was even lower in patients with lymph node metastasis (Fig. 2D, p=0.0029), 
Table I. Biological processes enriched by PEG10 elevation.

\begin{tabular}{|c|c|c|c|c|c|c|}
\hline No. & GS Details & Size & ES & NES & NOM p-val & FDR q-val \\
\hline 1 & MRNA_METABOLIC_PROCESS & 56 & 0.58 & 2.13 & 0 & 0.016 \\
\hline 2 & MRNA_PROCESSING_GO_0006397 & 45 & 0.62 & 2.12 & 0 & 0.009 \\
\hline 3 & RNA_BINDING & 196 & 0.38 & 2.04 & 0.002 & 0.017 \\
\hline 4 & REGULATION_OF_CELL_CYCLE & 153 & 0.55 & 1.97 & 0 & 0.036 \\
\hline 5 & CELLULAR_PROTEIN_COMPLEX_ASSEMBLY & 28 & 0.53 & 1.92 & 0.002 & 0.059 \\
\hline 6 & CELL_CYCLE_GO_0007049 & 260 & 0.56 & 1.91 & 0.002 & 0.059 \\
\hline 7 & RIBONUCLEOPROTEIN_COMPLEX & 105 & 0.49 & 1.91 & 0.002 & 0.05 \\
\hline 8 & RNA_PROCESSING & 117 & 0.46 & 1.89 & 0.022 & 0.055 \\
\hline 9 & MITOTIC_CELL_CYCLE & 124 & 0.65 & 1.87 & 0.004 & 0.065 \\
\hline 10 & CHROMOSOME & 104 & 0.6 & 1.85 & 0.004 & 0.071 \\
\hline 11 & REGULATION_OF_MITOSIS & 34 & 0.75 & 1.84 & 0.002 & 0.075 \\
\hline 12 & CHROMOSOMAL_PART & 80 & 0.62 & 1.84 & 0.004 & 0.069 \\
\hline 13 & GUANYL_NUCLEOTIDE_BINDING & 42 & 0.49 & 1.83 & 0.004 & 0.073 \\
\hline 14 & GTP_BINDING & 42 & 0.49 & 1.83 & 0.004 & 0.068 \\
\hline 15 & CELL_CYCLE_PROCESS & 156 & 0.62 & 1.82 & 0.004 & 0.069 \\
\hline 16 & CELL_CYCLE_PHASE & 140 & 0.61 & 1.82 & 0.004 & 0.065 \\
\hline 17 & $\begin{array}{l}\text { REGULATION_OF_CYCLIN_DEPENDENT } \\
\text { PROTEIN_KINASE_ACTIVITY }\end{array}$ & 39 & 0.63 & 1.81 & 0.002 & 0.067 \\
\hline 18 & RIBONUCLEASE_ACTIVITY & 21 & 0.64 & 1.81 & 0 & 0.065 \\
\hline 19 & INTERPHASE & 58 & 0.57 & 1.81 & 0.004 & 0.063 \\
\hline 20 & REGULATION_OF_MITOTIC_CELL_CYCLE & 16 & 0.69 & 1.81 & 0.004 & 0.06 \\
\hline
\end{tabular}

Statistical data were analyzed by GSEA software. ES, enrichment score; NES, normal enrichment score; NOM p-val, normal p-value; FDR q-val, false discovery rate q-value.

while there was no significant difference in PEG10 high and low group in non-metastatic patients (Fig. $2 \mathrm{E}, \mathrm{p}=0.24$ ). These results indicated that PEG10 is negatively correlated with clinical outcome.

PEG10 enhanced cell cycle processes in breast cancer. GSE3494 dataset was used to analyze PEG10 relationship with biological processes by GSEA. The gene profile of high PEG10 expression group (top 25\%) and low PEG10 expression group (75\%) were entered to the GSEA software and GMT file C5 (GO gene sets) was selected to process the analysis. The first twenty relevant biological processes that had $p<0.05$ and false discovery rate $\mathrm{p}<0.08$ were selected and are shown in Table I with enrichment score, normalized enrichment score, normal p-value and false discovery rate values. Fig. 3 shows gene set differences in PEG10 high vs. low patients, indicating that PEG10 regulates gene sets mainly associated with cell cycle progression.

PEG10 accelerates breast cancer cell proliferation and clone formation. First, we constructed PEG10 overexpression plasmid PEG10-EGFP. MDA-MB-231 cells were transfected with PEG10-EGFP plasmid or Control-EGFP plasmid, and mRNA and protein were collected after $48 \mathrm{~h}$ of transfection. Real-time quantitative PCR and western blotting were performed for PEG10 expression at mRNA level
(Fig. 4A) and protein level (Fig. 4B), respectively. To assess the effect of proliferation by PEG10, MDA-MB-231 cells were transfected with plasmids for $48 \mathrm{~h}$ and then seeded in culture plates. Cell viability was measured by CCK-8 assay, and results showed that overexpressed PEG10 promoted the proliferation rates of MDA-MB-231 cells compared with the control group in a time-dependent manner (Fig. 4C). In addition, clone formation assay also presented similar results (Fig. 4D) in which clone spheres were enlarged after the PEG10 elevation (Fig. 4E). All results gathered illustrated that PEG10 could enhance breast cancer cell proliferation and clone formation.

PEG10 facilitated MDA-MB-231 cells into $S$ and $G 2 / M$ phase. Flow cytometry analysis revealed that the percentage of MDA-MB-231/PEG10 transfect cells was higher in the $\mathrm{S}$ phase and G2/M phase, while the proportion was lower in the G0/G1 phase of the MDA-MB-231/PEG10 transfected cells as compared to the control group, as shown in Fig. 5A and B. These data provided evidence that PEG10 facilitated the cell cycle progression of MDA-MB-231 cells. Fig. 5C shows the quantitative statistical data of the cell cycle.

Overexpression of PEG10 promotes breast cancer cell migration and invasion. To investigate the migration ability initiated by PEG10, would healing assay were performed 
A

$$
\text { Regulation of cell cycle }
$$$$
\mathrm{p}=0.000 \quad \mathrm{FDR}=0.036
$$

Enrichment plot: REGULATION_OF_CELL_CYCLE

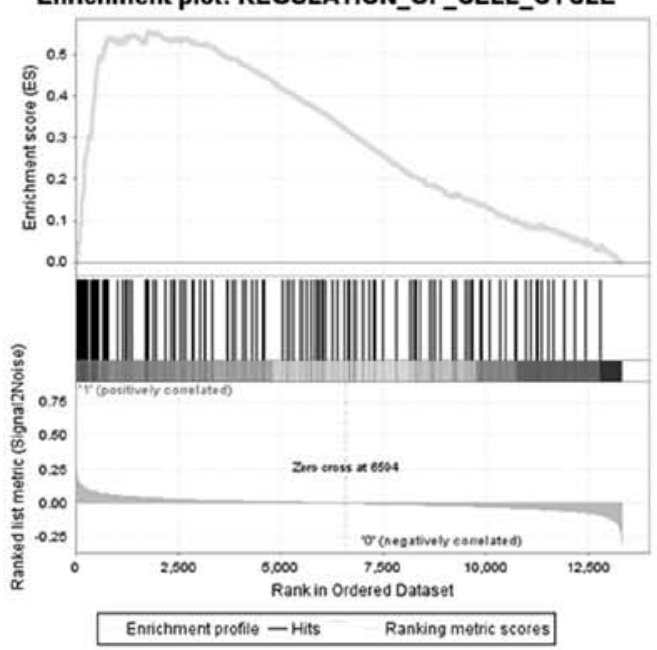

C

Mitotic cell cycle $\mathrm{p}=0.004 \quad \mathrm{FDR}=0.065$

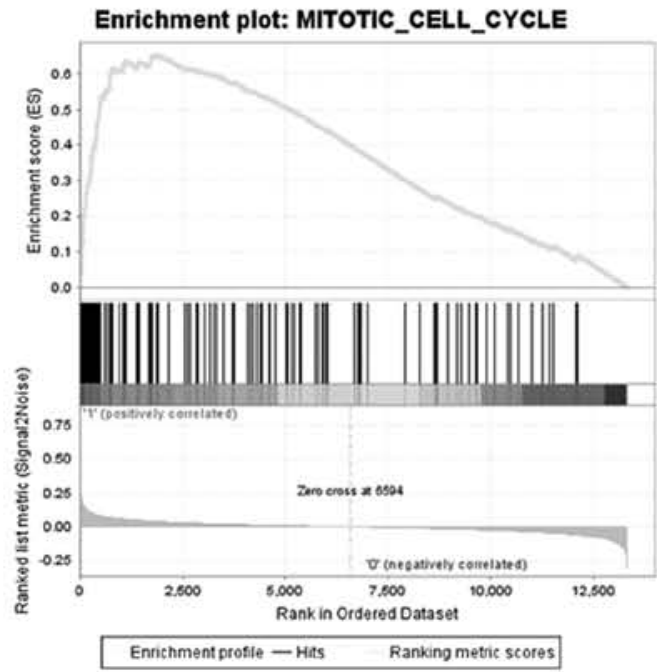

E

Cell cycle phase $\mathrm{p}=0.004 \quad \mathrm{FDR}=0.065$

Enrichment plot: CELL_CYCLE_PHASE

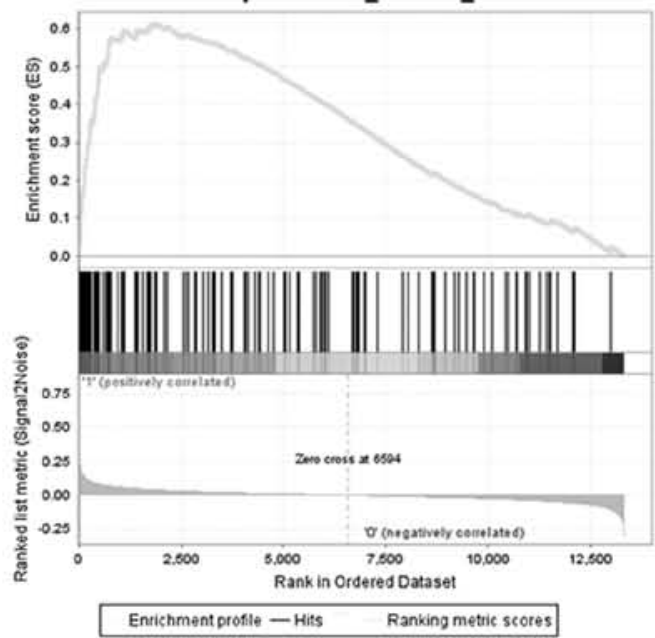

B

Cell cycle GO-0007049

$$
\mathrm{p}=0.002 \quad \mathrm{FDR}=0.059
$$

Enrichment plot: CELL_CYCLE_GO_0007049

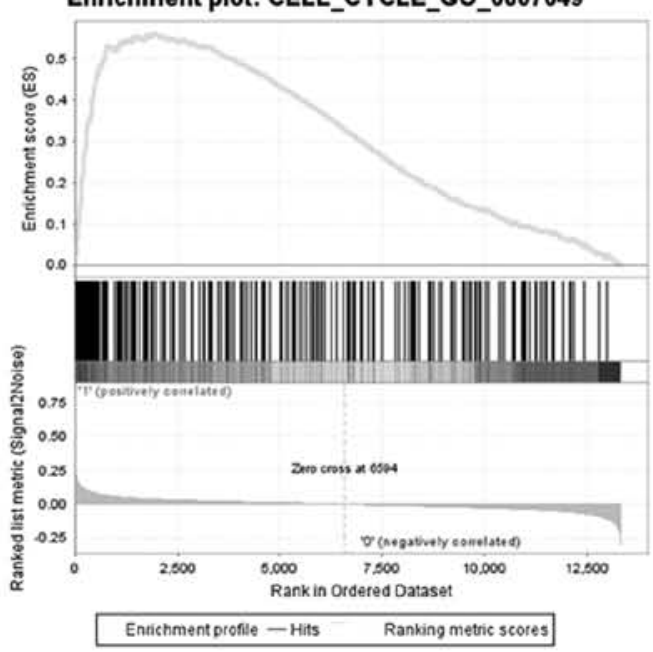

D

Cell cycle process $\mathrm{p}=0.004 \quad \mathrm{FDR}=0.069$

Enrichment plot: CELL_CYCLE_PROCESS



Entichment profile - Hits Ranking metric scotes

F Regulation of cyclin dependent protein kinase activity $\mathrm{p}=0.002 \quad$ FDR $=0.067$ Enrichment plot:

REGULATION_OF_CYCLIN_DEPENDENT_PROTEIN_KINA SE_ACTIVITY

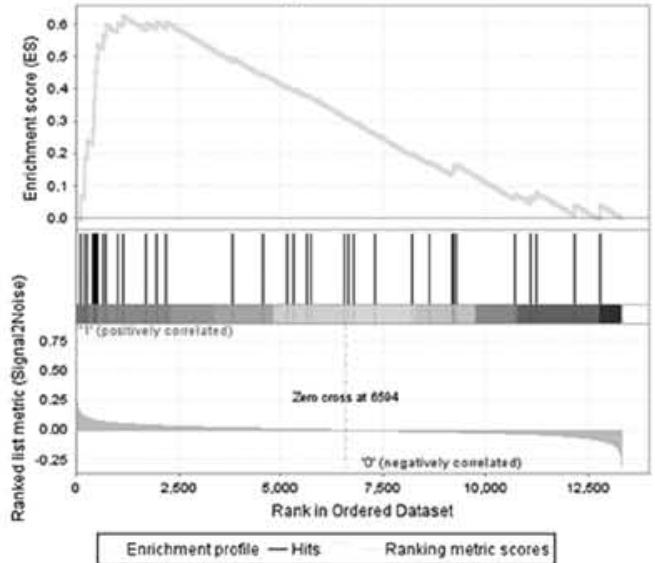

Figure 3. PEG10 enhances cell cycle process in breast cancer. Gene set enrichment analysis was determined by GSEA using GSE3494 dataset. The gene profile of high PEG10 expression groups (top 25\%) and low PEG10 expression groups (75\%) were entered to the GSEA software and GMT file C5 (GO gene set) was selected to process the analysis. 
A

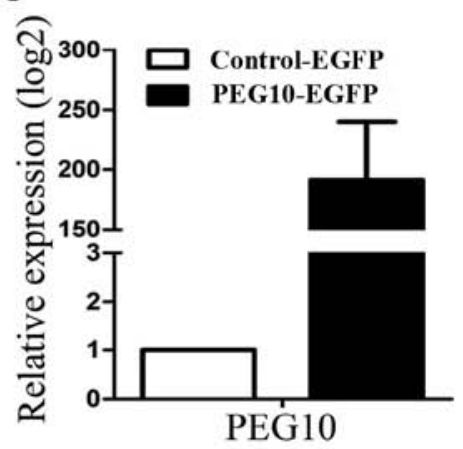

B

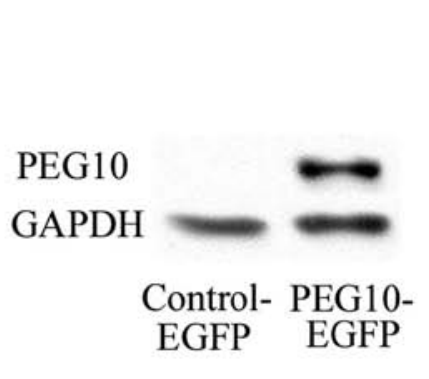

C

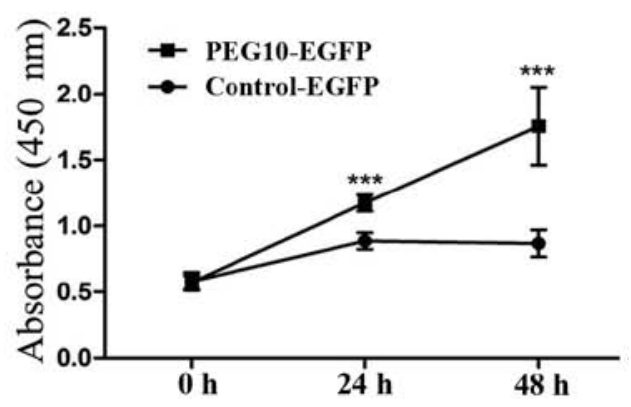

D

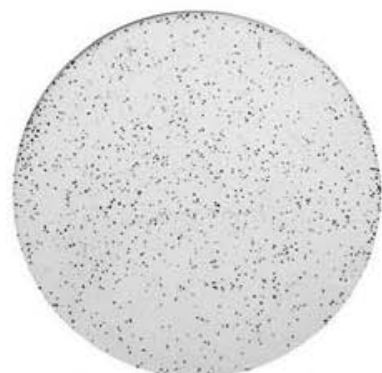

Control-EGFP

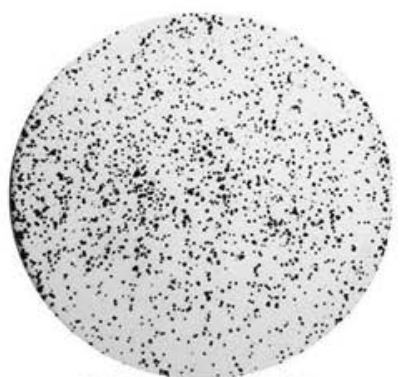

PEG10-EGFP

E

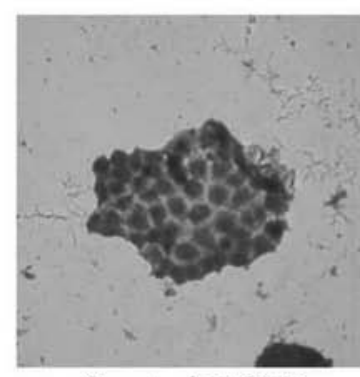

Control-EGFP

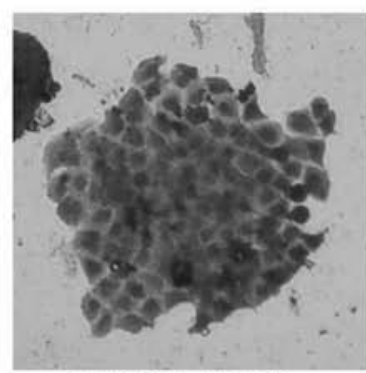

PEG10-EGFP

Figure 4. PEG10 accelerated breast cancer cell proliferation and clone formation. The expression of PEG10 was detected in mRNA (A) and protein level (B) after $48 \mathrm{~h}$ transfected with PEG10-EGFP and Control-EGFP. Proliferation was measured by CCK-8 assay at indicated times after transfected with PEG10EGFP and Control-EGFP for $48 \mathrm{~h}(\mathrm{C})$. Clone formation (D) and the clone sphere (E) were gained after transfected cells were seeded and incubated for 10 days. Each experiment was done in triplicate and results are shown as the mean value $\left({ }^{* * * *} \mathrm{p}<0.001\right)$.

A

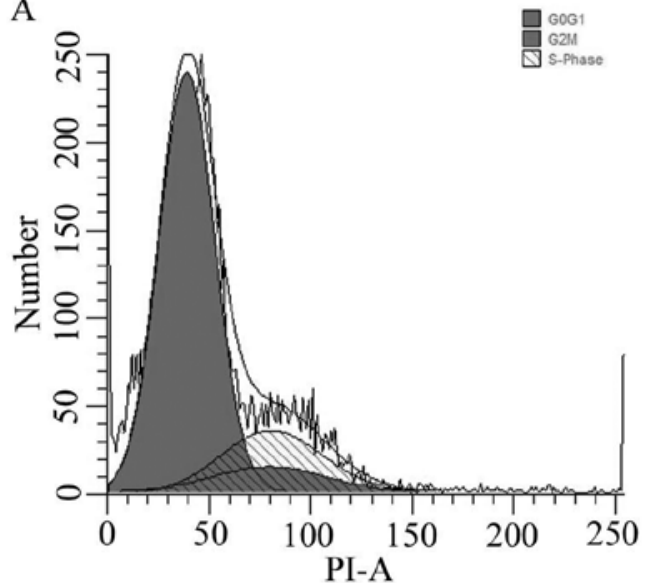

B

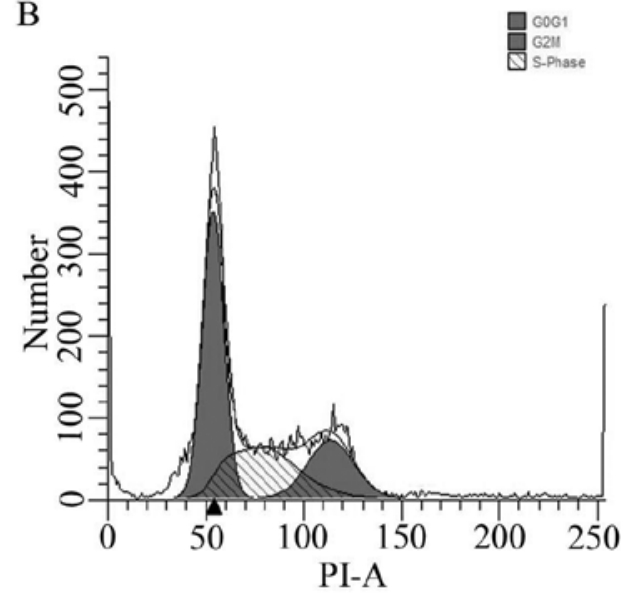

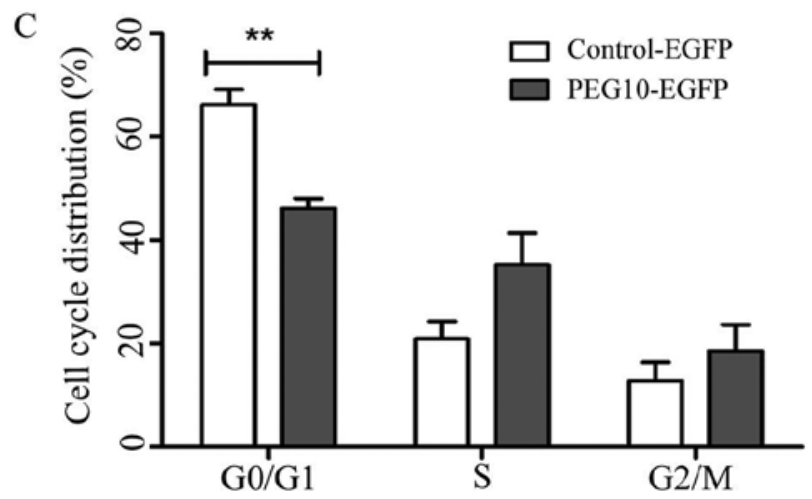

Figure 5. PEG10 facilitates MDA-MB-231 cells into S and G2/M phase. After $48 \mathrm{~h}$ of transfection, PI staining intensity of Control-EGFP (A) and PEG10EGFP (B) were detected by flow cytometry and results were analyzed by ModFit software. Quantitative statistical data of the cell cycle from A and B (C). The experiments were done in triplicate and results are shown as the mean value $\left({ }^{* *} \mathrm{p}<0.005\right)$. 
A
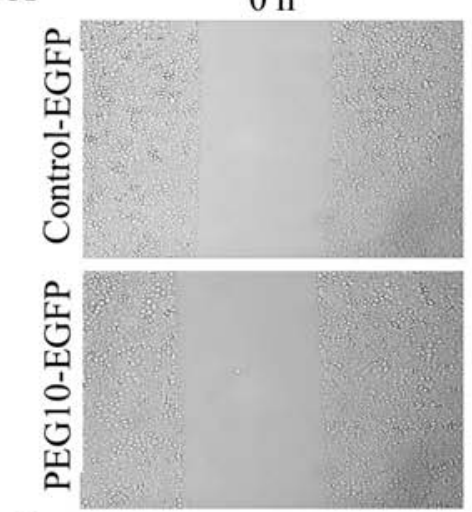

B



C






PEG10-EGFP



PEG10-EGFP
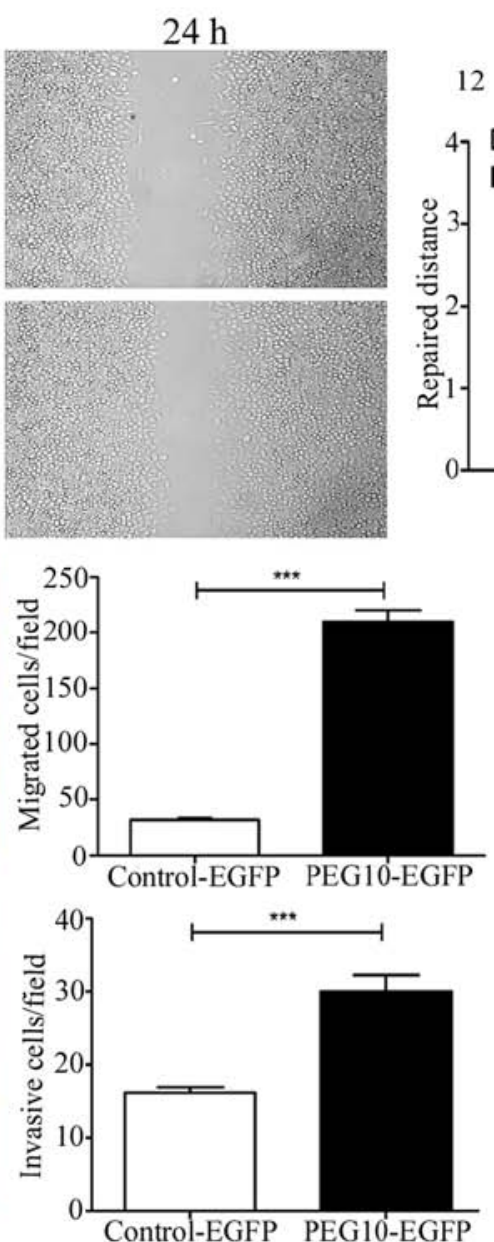

12

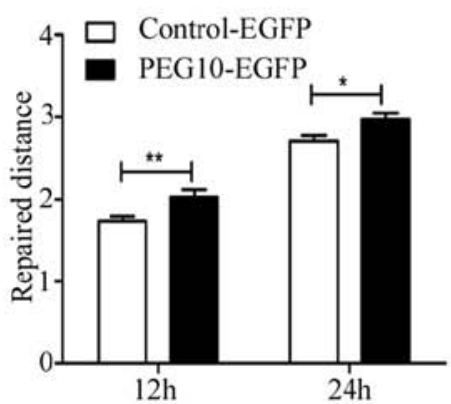

D

Figure 6. Overexpression of PEG10 promotes breast cancer cell migration and invasion. After cells were transfected with plasmid for $24 \mathrm{~h}$ and starved for $24 \mathrm{~h}$, wounds were scratched and images were obtained at each time point (A). Transfected cells were trans-migrated from transwell chamber with (B) or without (C) Matrigel, and cells in the lower membrane were dyed with crystal violet and images were tahen by microscopy. The expression levels of MMPs and other molecules were analyzed after transfection (D). Each experiment was done in triplicate and statistical data are shown as the mean value $\left({ }^{*} \mathrm{p}<0.05,{ }^{* *} \mathrm{p}<0.005\right.$, $\left.{ }^{* * * *} \mathrm{p}<0.001\right)$.

in MDA-MB-231 cells. Fig. 6A demonstrates that PEG10 promoted MDA-MB-231 cells to migrate. Transwell assay (Fig. 6B) showed a similar result that PEG10 overexpressing cells migrated more than the control group. Furthermore, Matrigel invasion assay (Fig. 6C) also suggested that PEG10 was involved in breast cancer cells invasion. After overexpressing PEG10 levels in breast cancer, MMP-1, MMP-2 and MMP-9, which function as matrix decomposers, were evidently increased, while TIMP-1 and TIMP-2 decreased (Fig. 6D). Importantly, the vital cell-to-cell junction molecule E-cadherin was downregulated after PEG10 overexpression (Fig. 6D), suggesting that PEG10 might influence the detachment and movement of cancer cells from primary sites to invade secondary sites.

\section{Discussion}

Breast cancer is frequently lethal with the second highest mortality rate $(15 \%)$ among women in America $(1,23,24)$. There were 64,640 new diagnosed in situ breast cancer cases, 232,340 invasive cases and 39,620 deaths due to breast cancer among all ages in the United States in 2013. One in every eight women will develop breast cancer, and invasive incidence is much higher in ages over 50, and so is the mortality incidence (25). Breast cancer with metastasis is devastating and effective treatments options are facing numerous challenges. Hence, efficient specific tumor-related genes, which could be used in the early diagnosis, prognosis and treatment of breast cancer are urgently required.

PEG10 was initially identified in 2001, and since then, it has been continuously positively associated with many types of cancers, including leukemia $(15,19,20)$, lymphoma (21), prostate cancer (12), liver cancer $(13,14)$, and lung cancer (22). Our recent report unveiled the role of PEG10 in Raji cell apoptosis resistance, proliferation, adhesion, migration and invasion (21). In addition, we demonstrated that PEG10 is associated with lung cancer progression and enhanced proliferation, carcinogenesis, migration and invasion ability of A549 cells (22). In this study, we investigated the relationship between PEG10 and breast cancer progression by bioinformatics analysis and the function of PEG10 in breast cancer cells proliferation, cell cycle, clone formation, migration and invasion by in vitro experiments.

With the development of genomics and computer sciences, bioinformatics analysis is emerging as a tool to evaluate and predict diseases and it is widely applied in research (26-28). For bioinformatics analysis, a large cohorts of samples $(n>100)$ 
with sufficient clinical information and follow-up records are used as the selecting criteria and GSE42568 $(n=121)$, GSE3494 $(n=251)$, GSE4922 ( $n=289)$, GSE10780 $(n=185)$ were chosen in this study. Our results showed that PEG10 was highly expressed in breast cancer tissues compared to normal breast tissues and highly expressed in metastasis tissues versus the non-metastatic tissues. At the same time, PEG10 expression was also linked with tumor grades and tumor size. Univariate survival analysis indicated that patients with PEG10 highexpression were more vulnerable to breast cancer. GSEA results showed that elevated PEG10 expression was closely related with cell cycle processes. These results confirmed that PEG10 may be important to breast cancer development and could be used as a prognosis indicator. In addition, the GSEA table results not only demonstrated that PEG10 was highly related to cell cycle, but also revealed that PEG10 may influence the mRNA metabolic process, cellular protein complex assembling and other biological processes.

For better understanding the role of PEG10 in breast cancer, we performed cytology experiments to verify the results we obtained from GEO datasets. We constructed a PEG10 fulllength plasmid and transfected it to MDA-MB-231 cells. Overexpression of PEG10 was observed to strongly enhance cell proliferation, clone formation ability, and the clone size was much bigger than in the control group. PEG10 also promoted cells into $\mathrm{S}$ and $\mathrm{G} 2 / \mathrm{M}$ phases, which was consisted with GSEA results. In addition, PEG10 overexpressing cells exhibited enhanced migration and invasion ability based on wound healing assay and invasion assay. Altogether, we propose that PEG10 is strongly associated with breast cancer development and progression.

As tumors progress to higher pathological grades of malignancy, they exhibit invasive properties, such as decreased attachment to other cells. E-cadherin has been seen as a key cell-to-cell adhesion molecule, and its loss has been associated with weakened maintenance of adherens junctions $(29,30)$. In our study, we observed that E-cadherin expression was reduced by PEG10 overexpression, indicating the occurrence of weakened cell junction and the possibility of migration. The process of cancer invasion requires extracellular matrix degradation, which is orchestrated by MMPs and TIMPs (31-33). It has been widely reported that MMP-2 exhibited a vital role in invasion of breast cancer (34-37). MMP-2 is highly expressed in breast cancer and is also positively related to shortened survival (38). Siegel et al reported that in breast cancer invasion, not only MMP-2 and MMP-9 expression was enhanced, but also MMP-1 and MMP-13 were upregulated in a CCR9dependent fashion (1). Therefore, in this study we also showed that MMP-1, MMP-2 and MMP-9 were upregulated while TIMP-1 and TIMP-2 were downregulated due to PEG10 overexpression. These results confirmed that PEG10 could enhance the migration and invasion ability of breast cancer. However, the expression of Twist, one of the most important molecules regulating cancer metastasis $(39,40)$, was not significant (data not shown), suggesting that PEG10 effect on migration may not be influenced by Twist signaling.

Taken together, this study elucidated that PEG10 was highly expressed in breast cancer and positively correlated with clinicopathological features. Furthermore, we verified that overexpressing PEG10 could promote breast cancer cell proliferation, clone formation, cell cycle, migration and invasion. These findings may be beneficial to a better understanding of breast cancer and to shed light on potential targets for breast cancer diagnosis, and therapeutics.

\section{Acknowledgements}

This study was supported by the National Natural Science Foundation of China (nos. 81400121, 81270607, 81541027 and 81501352).

\section{References}

1. Siegel RL, Miller KD and Jemal A: Cancer statistics, 2015. CA Cancer J Clin 65: 5-29, 2015.

2. Ricceri F, Fasanelli F, Giraudo MT, Sieri S, Tumino R, Mattiello A, Vagliano L, Masala G, Quirós JR, Travier N, et al: Risk of second primary malignancies in women with breast cancer: Results from the European prospective investigation into cancer and nutrition (EPIC). Int J Cancer 137: 940-948, 2015.

3. Cappello P, Blaser H, Gorrini C, Lin DC, Elia AJ, Wakeham A, Haider S, Boutros PC, Mason JM, Miller NA, et al: Role of Nek2 on centrosome duplication and aneuploidy in breast cancer cells. Oncogene 33: 2375-2384, 2014.

4. Bush TL, Payton M, Heller S, Chung G, Hanestad K, Rottman JB, Loberg R, Friberg G, Kendall RL, Saffran D, et al: AMG 900, a small-molecule inhibitor of aurora kinases, potentiates the activity of microtubule-targeting agents in human metastatic breast cancer models. Mol Cancer Ther 12: 2356-2366, 2013.

5. Wang Q, Wei J, Su P and Gao P: Histone demethylase JARID1C promotes breast cancer metastasis cells via down regulating BRMS1 expression. Biochem Biophys Res Commun 464: 659-666, 2015

6. Lee M, Beggs SM, Gildea D, Bupp S, Lichtenberg J, Trivedi NS $\mathrm{Hu}$ Y, Bodine DM and Crawford NP; NISC Comparative Sequencing Program: Necdin is a breast cancer metastasis suppressor that regulates the transcription of c-Myc. Oncotarget 6: 31557-31568, 2015.

7. Johnson-Holiday C, Singh R, Johnson E, Singh S, Stockard CR, Grizzle WE and Lillard JW Jr: CCL25 mediates migration, invasion and matrix metalloproteinase expression by breast cancer cells in a CCR9-dependent fashion. Int J Oncol 38: 1279-1285, 2011.

8. Ooms LM, Binge LC, Davies EM, Rahman P, Conway JR, Gurung R, Ferguson DT, Papa A, Fedele CG, Vieusseux JL, et al: The inositol polyphosphate 5-phosphatase PIPP regulates AKT1-dependent breast cancer growth and metastasis. Cancer Cell 28: 155-169, 2015.

9. Mahauad-Fernandez WD, DeMali KA, Olivier AK and Okeoma CM: Bone marrow stromal antigen 2 expressed in cancer cells promotes mammary tumor growth and metastasis. Breast Cancer Res 16: 493, 2014.

10. Li L, Liu B, Zhang X and Ye L: The oncoprotein HBXIP promotes migration of breast cancer cells via GCN5-mediated microtubule acetylation. Biochem Biophys Res Commun 458: 720-725, 2015.

11. Ono R, Kobayashi S, Wagatsuma H, Aisaka K, Kohda T, KanekoIshino T and Ishino F: A retrotransposon-derived gene, PEG10, is a novel imprinted gene located on human chromosome 7q21. Genomics 73: 232-237, 2001.

12. Akamatsu S, Wyatt AW, Lin D, Lysakowski S, Zhang F, Kim S, Tse C, Wang K, Mo F, Haegert A, et al: The placental gene PEG10 promotes progression of neuroendocrine prostate cancer. Cell Rep 12: 922-936, 2015.

13. Tsou AP, Chuang YC, Su JY, Yang CW, Liao YL, Liu WK, Chiu JH and Chou CK: Overexpression of a novel imprinted gene, PEG10, in human hepatocellular carcinoma and in regenerating mouse livers. J Biomed Sci 10: 625-635, 2003.

14. Bang H, Ha SY, Hwang SH and Park CK: Expression of PEG10 is associated with poor survival and tumor recurrence in hepatocellular carcinoma. Cancer Res Treat 47: 844-852, 2015.

15. Kainz B, Shehata M, Bilban M, Kienle D, Heintel D, KrömerHolzinger E, Le T, Kröber A, Heller G, Schwarzinger I, et al: Overexpression of the paternally expressed gene 10 (PEG10) from the imprinted locus on chromosome 7q21 in high-risk B-cell chronic lymphocytic leukemia. Int J Cancer 121: 1984-1993, 2007. 
16. Ono R, Nakamura $K$, Inoue $K$, Naruse $M$, Usami $T$, Wakisaka-Saito N, Hino T, Suzuki-Migishima R, Ogonuki N, Miki H, et al: Deletion of Peg10, an imprinted gene acquired from a retrotransposon, causes early embryonic lethality. Nat Genet 38: 101-106, 2006.

17. Liu Z, Yang Z, Liu D, Li D, Zou Q, Yuan Y, Li J, Liang L, Chen M and Chen S: TSG101 and PEG10 are prognostic markers in squamous cell/adenosquamous carcinomas and adenocarcinoma of the gallbladder. Oncol Lett 7: 1128-1138, 2014.

18. Li CM, Margolin AA, Salas M, Memeo L, Mansukhani M, Hibshoosh H, Szabolcs M, Klinakis A and Tycko B: PEG10 is a c-MYC target gene in cancer cells. Cancer Res 66: 665-672, 2006.

19. Hu C, Xiong J, Zhang L, Huang B, Zhang Q, Li Q, Yang M, Wu Y, Wu Q, Shen Q, et al: PEG10 activation by co-stimulation of CXCR5 and CCR7 essentially contributes to resistance to apoptosis in $\mathrm{CD} 19^{+} \mathrm{CD} 34^{+} \mathrm{B}$ cells from patients with B cell lineage acute and chronic lymphocytic leukemia. Cell Mol Immunol 1: 280-294, 2004.

20. Chunsong H, Yuling H, Li W, Jie X, Gang Z, Qiuping Z, Qingping G, Kejian Z, Li Q, Chang AE, et al: CXC chemokine ligand 13 and $\mathrm{CC}$ chemokine ligand 19 cooperatively render resistance to apoptosis in $\mathrm{B}$ cell lineage acute and chronic lymphocytic leukemia $\mathrm{CD} 23^{+} \mathrm{CD} 5^{+} \mathrm{B}$ cells. J Immunol 177: 6713-6722, 2006.

21. Xiong J, Qin J, Zheng Y, Peng X, Luo Y and Meng X: PEG10 promotes the migration of human Burkitt's lymphoma cells by up-regulating the expression of matrix metalloproteinase- 2 and -9. Clin Invest Med 35: E117-E125, 2012.

22. Deng X, Hu Y, Ding Q, Han R, Guo Q, Qin J, Li J, Xiao R, Tian S, $\mathrm{Hu} \mathrm{W}$, et al: PEG10 plays a crucial role in human lung cancer proliferation, progression, prognosis and metastasis. Oncol Rep 32: 2159-2167, 2014

23. Vila J, Gandini S and Gentilini O: Overall survival according to type of surgery in young ( $\leq 40$ years) early breast cancer patients: A systematic meta-analysis comparing breast-conserving surgery versus mastectomy. Breast 24: 175-181, 2015.

24. Mariz K, Ingolf JB, Daniel H, Teresa NJ and Erich-Franz S: The Wnt inhibitor dickkopf-1: A link between breast cancer and bone metastases. Clin Exp Metastasis 32: 857-866, 2015.

25. DeSantis C, Ma J, Bryan L and Jemal A: Breast cancer statistics, 2013. CA Cancer J Clin 64: 52-62, 2014.

26. Wu W, Morrissey CS, Keller CA, Mishra T,Pimkin M, Blobel GA Weiss MJ and Hardison RC: Dynamic shifts in occupancy by TAL1 are guided by GATA factors and drive large-scale reprogramming of gene expression during hematopoiesis. Genome Res 24: 1945-1962, 2014.

27. Wilson NK, Kent DG, Buettner F, Shehata M, Macaulay IC, Calero-Nieto FJ, Sánchez Castillo M, Oedekoven CA, Diamanti E, Schulte R, et al: Combined single-cell functional and gene expression analysis resolves heterogeneity within stem cell populations. Cell Stem Cell 16: 712-724, 2015.
28. Shirai CL, Ley JN, White BS, Kim S, Tibbitts J, Shao J, Ndonwi M, Wadugu B, Duncavage EJ, Okeyo-Owuor T, et al: Mutant U2AF1 expression alters hematopoiesis and pre-mRNA splicing in vivo. Cancer Cell 27: 631-643, 2015.

29. Hanahan D and Weinberg RA: Hallmarks of cancer: The next generation. Cell 144: 646-674, 2011.

30. Piao HL, Yuan Y, Wang M, Sun Y, Liang H and Ma L: $\alpha$-catenin acts as a tumour suppressor in E-cadherin-negative basal-like breast cancer by inhibiting NF- $\kappa \mathrm{B}$ signalling. Nat Cell Biol 16: 245-254, 2014.

31. Waleh NS, Murphy BJ and Zaveri NT: Increase in tissue inhibitor of metalloproteinase-2 (TIMP-2) levels and inhibition of MMP-2 activity in a metastatic breast cancer cell line by an anti-invasive small molecule SR13179. Cancer Lett 289: 111-118, 2010.

32. Lee S, Desai KK, Iczkowski KA, Newcomer RG, Wu KJ, Zhao YG, Tan WW, Roycik MD and Sang QX: Coordinated peak expression of MMP-26 and TIMP-4 in preinvasive human prostate tumor. Cell Res 16: 750-758, 2006.

33. Nagase H: Cell surface activation of progelatinase A (proMMP-2) and cell migration. Cell Res 8: 179-186, 1998

34. Vizoso FJ, González LO, Corte MD, Rodríguez JC, Vázquez J, Lamelas ML, Junquera S, Merino AM and García-Muñiz JL: Study of matrix metalloproteinases and their inhibitors in breast cancer. Br J Cancer 96: 903-911, 2007.

35. Jacob A, Jing J, Lee J, Schedin P, Gilbert SM, Peden AA, Junutula JR and Prekeris R: Rab40b regulates trafficking of MMP2 and MMP9 during invadopodia formation and invasion of breast cancer cells. J Cell Sci 126: 4647-4658, 2013.

36. Kambach DM, Sodi VL, Lelkes PI, Azizkhan-Clifford J and Reginato MJ: ErbB2, FoxM1 and 14-3-3 $\zeta$ prime breast cancer cells for invasion in response to ionizing radiation. Oncogene 33: 589-598, 2014.

37. Addison JB, Koontz C, Fugett JH, Creighton CJ, Chen D, Farrugia MK, Padon RR, Voronkova MA, McLaughlin SL, Livengood RH, et al: KAP1 promotes proliferation and metastatic progression of breast cancer cells. Cancer Res 75: 344-355, 2015.

38. Talvensaari-Mattila A,PääkköP,Höyhtyä M,Blanco-Sequeiros G and Turpeenniemi-Hujanen T: Matrix metalloproteinase-2 immunoreactive protein: A marker of aggressiveness in breast carcinoma. Cancer 83: 1153-1162, 1998.

39. Shi J, Wang Y, Zeng L, Wu Y, Deng J, Zhang Q, Lin Y, Li J, Kang T, Tao M, et al: Disrupting the interaction of BRD4 with diacetylated Twist suppresses tumorigenesis in basal-like breast cancer. Cancer Cell 25: 210-225, 2014.

40. Fu J, Qin L, He T, Qin J, Hong J, Wong J, Liao L and Xu J: The TWIST/Mi2/NuRD protein complex and its essential role in cancer metastasis. Cell Res 21: 275-289, 2011. 